\title{
Genetic evidence of spotted fever group rickettsiae in ticks collected from domestic animals in Eastern Cape, South Africa.
}

\author{
BENSON IWERIEBOR ${ }^{1}$, Ayabulela Nqoro ${ }^{2}$, Anthony $\mathrm{Okoh}^{3}$, and Larry $\mathrm{Obi}^{1}$ \\ ${ }^{1}$ Sefako Makgatho Health Sciences University \\ ${ }^{2}$ University of Fort Hare Faculty of Science and Agriculture \\ ${ }^{3}$ University of Fort Hare
}

May 6, 2020

\begin{abstract}
Background: Ticks have the propensity to transmit plethora of pathogens that have zoonotic potentials. Their distribution, diversity and the pathogens they transmit differs from one ecological location to another. SFG (spotted fever group) rickettsiae which are predominantly transmitted by different Ixodes ticks are responsible for emerging zoonotic diseases globally. Ticks were collected from domesticated animals in Raymond Nkandla Municipality, Eastern Cape, South Africa. The ticks were identified morphologically prior to DNA extraction and were molecularly identified by randomly selecting ticks from the morphologically delineated groups. To assess for the presence of tick-borne pathogens belonging to Rickettsia spp. by PCR (polymerase chain reaction) we used specific primer pairs targeting the gltA, ompA and ompB genes. The selected amplified ticks (according to morphological delineations), all positive ompB and forty three ompA amplicons were sequenced in a commercial sequencing facility. The obtained nucleotide sequences were edited and subjected to BLASTn for homology search and phylogenetic analyses were performed with MEGA 7 Version for evolutionary relationships with curated reference sequences in GenBank. Results: A total of 953 ticks collected in the study were delineated into three genera consisting of Amblyomma, Rhipicephalus and Hyalomma in decreasing order of abundance. The presence of Rickettsia DNA was detected in 60/953 (6.3\%) from the three genera of ticks screened. Genetic analyses of the DNA sequences obtained showed that they have phylogenetic relationship to members of spotted fever group rickettsiae with R. africae, R. parkeri and R.tamurae being the SFGR (spotted fever group rickettsiae) detected in the screened ticks. Conclusion: This report shows that $\mathrm{R}$. africae is the predominant spotted fever group rickettsiae in ticks collected from domestic animals in the study area and the human health impacts are not known.
\end{abstract}

\section{INTRODUCTION}

Tick-borne pathogens have been identified as the etiologic agents of emerging important human diseases especially in many tropical countries in Africa, Asia and South America (Parola et al., 2013). In many subSaharan Africa countries, these diseases are rampant in rural communities where there are frequent contacts between humans and domestic animals that are hosts to these ticks (Parola et al., 2013; Raoult and Roux, 1997). Rickettsioses are zoonotic diseases that are caused by some pathogenic Rickettsia spp. They are one of the oldest known vector-borne zoonotic diseases whose severity varies from one etiologic agent to another. There are many recognized species of Rickettsia that are delineated into four major groups namely; the typhus group consisting of two species which are R.typhi andR.prowazekii, the spotted fever group which contains many species that are wholly transmitted to humans through ticks bite, the transitional group comprising of R.australis, $R$. akari and R.felis that are associated with ticks, mites and fleas and the ancestral group made up of R.bellii and R.canadensis. Beside these groups, there are also many otherRickettsia species that do exist but have not been fully characterized (Raoult and Roux, 1997; Bogovic et al., 2016). The current guidelines for the classification, delineation and description of novel rickettsial isolates are based on the 16S rRNA gene, the differences in nucleotide sequence of glt A, omp A, omp B, and the D genes 
that encodes for essential proteins in the organisms ( Portillo et al., 2017). The global distribution of tickborne rickettsioses varies from one region to the other as their geographical spread are determined by their ticks vectors whose distributions are generally governed by suitable environmental conditions like relative temperatures, humidity and biotopes which varies from one region to the other (Socolovschi et al., 2009; Tomassone et al., 2018).

Members of the genera Amblyomma, Hyalomma, Rhipicephalus, Ixodes, Dermacentor and Haemaphysalis are the species of tick that are generally involved in the transmission of tick-borne rickettsioses in the tropical regions of the world. Transmission of tick-borne rickettsioses could be either transstadial or transovarial transmission thus making some tick species such as Amblyomma to be a known reservoir of $R$. africae (Tomassone et al., 2018). In the Eastern Cape of South Africa, the most common species of ticks are members of the genera; - Amblyomma, Rhipicephalus, Haemaphysalis, and Hyalomma (Iweriebor et al., 2017; Yawa et al., 2019) and all these ticks' genera have the ability to transmit one or multitudes of zoonotic pathogens. While most tick-borne pathogens are linked to known tick vectors, it is possible in some cases for an etiologic agent of a particular disease to be indeterminate.

Members of the SFG of the genus Rickettsia are the etiologic agents of tick-borne rickettsioses a group of diseases that its ticks vectors and clinical severity varies from one geographic location to another (Tomassone et al., 2018). The epidemiological and clinical attributes of tick-borne diseases are tied to the ecology and biotopes of their tick vectors (Tomassone et al., 2018) as each of these diseases are confined to different geographical regions though their distribution and redistribution keep varying overtime as well as the species of ticks that transmit them. Prior to the development of molecular approaches which are highly selective and sensitive in disease diagnoses, severalRickettsia spp. had been detected in ticks but their roles in the etiology of disease in humans were unrecognized. However, in recent times such species that were previously thought innocuous have now been directly linked to the etiology of human diseases (Parola et al., 2005; Bogovic et al., 2016).

The Eastern Cape of South Africa is predominantly rural with intensive animal husbandry where the animals are kept in close proximity to homes. Couple with this is that there are many game reserves where these animals are in close contact with those in the wild. There exist great possibilities of these domesticated animals being infested with ticks from those originating from the wild thus making the spread of zoonotic pathogens possible in these localities. Also, with the very high prevalence of HIV/AIDS in these rural communities, the chances of these immunocompromised patients coming down with zoonotic infections are equally very probable as they lack the competent immune system that ordinarily fights off these infections in healthy folks. Besides, most of the SFGR infections present with symptoms that are very much similar to flu and could thus be misdiagnosed by clinicians who might not consider them as top priority in diagnoses and treatments due to lack of laboratory diagnosis prior to commencement of treatments. This study therefore, was aimed at epidemiological surveillance for tick-borneRickettsia pathogens in ticks collected from domesticated animals in communities which are close to natural game reserves and human habitations in order to assess the likelihood of zoonotic diseases in humans who might be infested with ticks.

\section{Materials and Methods}

\section{Ethical clearance}

The University of Fort Hare Ethics Committee granted the ethical clearance (REC-270710-02-RA) before the commencement of the study. Permission was granted by the farmers before any ticks collection could commence from their animals. Ticks collection was done with help of veterinary personnel and animal health technicians responsible for handling and treating the animals. 


\section{Study area, tick collection and identifications.}

The study was conducted from March 2017 to September 2018 in Raymond Mhlaba local Municipality in the Eastern Cape Province. Ticks collection sites and their geographical coordinates are as follow; from Debe, with the following coordinates: $32.836^{\circ} \mathrm{S} 27.154^{\circ} \mathrm{E}$, under Raymond Mhlaba local Municipality and Fort Beaufort located at $32^{\circ} 47^{\prime} 0^{\prime \prime}$ S, $26^{\circ} 38^{\prime}$ 0" E. Debe Location shares boundary with Great fish natural reserve which boast of some wild animals like antelope, boars, giraffe, deer, and others. Study site map can be accessed through: - https://tse2.mm.bing.net/th?id=OIP.GvQ4IE1x4Slo9oTuUb9EBgHaEk\&pid =Api\&P=0\&w $=284 \& \mathrm{~h}=176$

\section{Ticks collection}

Ticks were collected manually from domestic animals (goats, sheep, cattle and horses) by using forceps into $50 \mathrm{~mL}$ Nalgene tubes that contained $70 \%$ ethanol taking precautionary measures to avoid mix-up of samples on the basis of animals and collection sites as tubes were properly labelled accordingly and were stored at $4^{\circ} \mathrm{C}$ until further processing (Iweriebor et al., 2017). All tick species collected were identified using morphological criteria and appropriate taxonomic keys and processed accordingly (Iweriebor et al., 2017; Yawa et al., 2019; Walker et al., 2003; Williamson et al., 2010). Ticks genders were not taken into consideration as it does not affect its ability to transmit pathogens

\section{Total genomic DNA extraction from ticks}

Prior to DNA extraction, the preserved specimens were washed with sterile distilled water and left to dry in a sterile Petri dish. This was done in order to remove all the ethanol residues from the tick samples which might negatively affect the downstream PCR reactions. The ticks were chopped into bits with sterile blades in the Petri dishes based on their morphological delineations and processed separately for DNA extraction using ReliaPrep DNA Tissue miniprep system ZYMORESEARCH Quick DNA Universal Kit according to the manufacturer's instruction and as previously described by Iweriebor et al. (2017). The adult engorged and non-engorged ticks were processed separately while the nymphs of the same species collected from the same animal were pooled together and processed for DNA extraction. All ticks processing and PCR reactions were performed in biosafety cabinet which were always left sterile overnight using UV light. Absolute precautionary measures were adopted to avoid cross contaminations of reagents, blades and instruments used throughout the process as well as pipettor sets were dedicated to specific functions, i.e., reagent dispensing, template isolation, PCR setup, and template handling. Commercially certified DNA/RNase free filter barrier tips were used to prevent aerosol contamination. PCR setup was performed far from the isolation materials, and reagent handling was separated both physically and temporally from templates.

\section{Molecular identification of tick and detection of bacteria in ticks}

Each tick specimen was assessed using PCR for both tick identification and detection of Rickettsia spp. Confirmation of tick species identification was done by PCR targeting the mitochondrial 12S rDNA gene from representatives of the genera hitherto identified morphologically (Yawa et al., 2016; Williamson et al., 2010). Screening forRickettsia spp. was performed using three of the primer pairs as indicated in Table 1 that are used for the species identification ofRickettsia pathogens as previously reported by Regnery et al. (1991) and Kollars and Kengluecha. (2001) in a PCR assay as adapted by Williamson et al., (2010)

Table 1. Primer sequences used in the molecular identification of ticks and Rickettsia spp

Primer name Gene Primer Sequence (5' to3') Amplicon bp TM Ref

Tick DNA

85F 12S TTAAGCTTTTCAGAGGAATTTGCTC 11054.0 Walker et al., 2003

225R 12S TTTWWGCTGCACCTTGACTTAA 52.7

Rickettsia spp. 
Rr.190 70P rompA ATGGCGAATATTTCTCCAAAA 610 52.5 Regnery et al., 1991

Rr.190 602N rompA AGTGCAGCATTCGCTCCCCCT 64.9

BG1-21 rompB GGCAATTAATATCGCTGACGG 511 55.6 Eremeeva \&Raoult, 1994

BG2-20 rompB GCATCTGCACTAGCACTTTC 55.2

RrCS372 gltA TTTGTAGCTCTTCTCATCCTATGGC 41059.0 Kollars and Kengluecha,2001

RrCS989 gltA CCAAGTTCCTTTAATACTTCTTTGC 57.4

\section{DNA sequencing, sequence editing and BLASTn search}

The amplified PCR products were sequenced in a commercial sequencing facility. Only 43 samples out of the 60 positive omp A amplicons were sequenced due to some technical constraints and all the positiveomp $\mathrm{B}$ amplicons were sequenced while all the amplified representatives of tick's $12 \mathrm{~S}$ rDNA amplicons were also sequenced and none was sequenced for the glt A positive amplicons. The inability to sequence the glt A amplicons was due to financial constraint coupled with the fact that the discriminatory power to delineateRickettsia spp. by the three amplified genes are equal and as such any of the genes could suffice for delineation of the species among the genus Rickettsia. The alignment and sequence editing was achieved using Geneious Prime 2019.0.3 version. The generated nucleotide sequences were subjected to homology search using the Basic Local Alignment Search Tool (National Center for Biotechnology Information, www.ncbi.nlm.nih.gov) where the edited sequences were compared with representative genetic sequences of previously characterized rickettsial and tick species curated in the GenBank NCBI. Rickettsial sequences that had above $97 \%$ homology with test samples were used as reference strains for phylogenetic analyses along with other curated representatives of SFGR sequences in the GenBank.

\subsubsection{Phylogenetic analysis}

Edited sequences were aligned with reference sequences obtained from GenBank after the BLAST search which was used to construct a maximum possible phylogenetic tree using Mega 7 version software with 1000 bootstrap replicate. Phylogenetic trees were constructed using BioEdit Tree Builder. Sequence data sets for positive samples were submitted to NCBI GenBank for accession numbers.

\section{RESULTS}

\section{Tick prevalence within the two study sites}

Nine hundred and fifty three (953) ticks' samples were collected from the designated localities in Raymond Mhlaba Local Municipality of Eastern Cape Province and analyzed for the prevalence of tick-borne rickettsial pathogens in the sampling sites.

Morphological identification method used to delineate the collected ticks showed that they belonged to three genera, which wereRhipicephalus, Amblyomma and Hyalomma with six different species. Amongst them were $A$. hebraeum $39.14 \%$ ( $\mathrm{n}=373$ adult), $R$. appendiculatus $16.4 \%$ ( $\mathrm{n}=156(34)$ adult), R. microplus $13.96 \%$ ( $\mathrm{n}=133$ adult), R. simus. $11.75 \%$ ( $\mathrm{n}=112$ adult), Rhipicephalus eversti eversti $9.02 \%$ ( $\mathrm{n}=86$ adult), and $H$. truncatum $9.76 \%$ ( $\mathrm{n}=93$ adult) in decreasing order of their prevalence. A. hebraeum was the most prevalent species in both study sites with cattle serving as the common host.

Furthermore, BLAST analysis of the edited tick 12S mitochondrial rDNA sequences showed that they all had more than $97 \%$ sequence homology with curated ticks' sequences in GenBank. Molecular identification of the representatives of different ticks delineated by morphological methods confirmed the accuracy of the morphologic classification as shown in Figure 1. The proportions of the different ticks collected in the two 
study sites are presented in Tables 2 while Table 3 is the list of reference sequences obtained from GenBank that were used in the phylogenetic analysis of the ticks' sequences generated in this study.

Table 2: Proportion and distribution of collected tick species in Debe Location and Fort Beaufort, geographical coordinates: $32.836^{\circ} \mathrm{S} 27.154^{\circ} \mathrm{E}$, coordinates $32^{\circ} 47^{\prime} 0^{\prime \prime} \mathrm{S}, 26^{\circ} 38^{\prime} 0^{\prime \prime} \mathrm{E}$.

\begin{tabular}{llll}
\hline & & & Number of ticks \\
Animal & Tick species & Developmental stage & Rickettsia positive \\
\hline Cattle & R. eversti eversti & Adult & 775 \\
& A. hebraeum & Adult & 17025 \\
& R. microplus & Adult & 27 \\
R. appendiculatus & Adult & 943 \\
& R. simus & Adult & 67 \\
& H. truncatum & Adult & 55 \\
Roat & Adult eversti eversti & Adult & 0 \\
& A. hebraeum & Nymph & 11312 \\
& R. microplus & Adult & 79 \\
& R. simus & Adult & 24 \\
& H. truncatum & Adult & 20 \\
Sheep & R. appendiculatus & Adult & 622 \\
& R. eversti eversti & Adult & 99 \\
& A. hebraeum & Adult & 654 \\
R. microplus & Adult & 27 \\
R. simus & Adult & 21 \\
Horse & H. truncatum & Adult & 18 \\
\hline
\end{tabular}

Table 3: Reference tick strains used in phylogenetic analysis

\begin{tabular}{|c|c|c|c|}
\hline Strain Accession Number & Species & Geographical origin & References \\
\hline KU284929 & A. trigrinum & Brazil & Lado et al., 2015 \\
\hline KU284920 & A. triste & Uruguay & Lado et al., 2015 \\
\hline KU284864 & A. parvitarsum & Argentina & Lado et al., 2015 \\
\hline KY676832 & R. annulatus & Israel & Duron, 2017 \\
\hline KY676839 & R. australis & South Africa & Duron, 2017 \\
\hline MF479198 & $R$. evertsi & DRC & Amzati et al., 2017 \\
\hline AF150043 & Boophilus & Jordan & Beati, 1999 \\
\hline EU921766 & R. microplus & Mozambique & Labruna et al., 2008 \\
\hline KU568502 & R. geigyi & Guinea-Bissau & Zuquete et al., 2016 \\
\hline MK332391 & R. microplus & Uganda & Ndekezi et al., 2018 \\
\hline MG076938 & A. maculatum & Mexico & Lado et al., 2017 \\
\hline KX377407 & A. gemma & Ethiopia & Kumsa, 2016 \\
\hline AF150049 & A. hebraeum & Zimbabwe & Beati, 1999 \\
\hline AF031865 & R. punctatus & Australia & Murrell et al., 1999 \\
\hline
\end{tabular}

\section{Detection, identification and prevalence ofRickettsia spp. in the study sites}

A total of $60(6.3 \%)$ positive samples for the three genes amplified were detected for Rickettsia spp. from the 953 ticks analyzed. PCR assays used for the identification of Rickettsia spp. were positive for all genes 
fragments profiled per every positive sample of which only some ompA (43) and all of omp B (60) amplicons were sequenced and analyzed to delineate the Rickettsia spp. down to the species level.

Rickettsial DNA fragments were obtained from the three genera of ticks collected in the study sites with more detected in A. hebreaum.Also, a higher proportion of the pathogens $(55 \%)$ were obtained in ticks collected from cattle, $23 \%$ prevalence in ticks from goats while that of sheep was $22 \%$.

A homology search for generated Rickettsia sequences showed that they had a high sequence similarity of above $97 \%$ with homologous sequences of Rickettsia in GenBank. Comparison of the 43omp A and 60 omp B sequences by BLAST analysis showed that majority of the sequences have [?]98\% homology with $R$. africaewhile only two samples (B188) and B209 had 99\% similarity with R. parkeri (KY113111) and 100\% homologous with $R$. tamurae (DQ113910) respectively based on $o m p$ B BLASTn analysis.However, discordances in homology between $o m p \mathrm{~A}$ and $о m p \mathrm{~B}$ sequences of the two samples were observed as shown in Figures 2a-d and 3a-d respectively. The omp A of sample 188 showed $99 \%$ similarity with Candidatus Rickettsia (EU272186) while the omp B of the same sample was 100\% homologous to R.parkeri (KY113111) in both amino acid and nucleotide sequences alignments. Similarly, A209 of sample 209 was $100 \%$ homologous with R. africae (EU622980) while B209 of the same sample was 100\% homologous with R.tamurae(DQ113910) respectively in both amino acid and nucleotide sequences alignments. Nucleotides and amino acids sequences alignments were performed with the corresponding homologous sequences of omp A andomp B sequences as shown in Figures 2A to D and 3A to D respectively.

Reference sequences used for phylogenetic analysis were randomly selected and analyzed with generated study sequences of omp A andomp B genes. The phylogenetic tree obtained for ompA gene showed that test sequences clustered with reference sequences from NCBI GenBank nucleotides database as illustrated in Figure 4. Majority of theomp A sequences clustered with R. africae (U43790; KJ645933, GU247115, MG515014) with bootstrap values above 81\% while sequences A196 and A198 clustered equidistance between R.tamurae(LC388791) R.africae (CP001612) with 92\% bootstrap reliability and A188 and A208 clustered with Candidatus Rickettsia EU272186 with $77 \%$ bootstrap value as shown in Figure 4 while the phylogenetic tree constructed with omp B sequences is shown in Figure 5 with bootstrap values above $70 \%$. Phylogenetic analyses of the omp A and omp B sequences support the observed discrepancies in homology. Sequence B150 clustered between R.rhipicephali(AF123719) and R.parkeri (KY113111) phylogenetically but a BLAST search showed it as having close homology with UnculturedRickettsia clone and $R$. conorii (FJ015092) by $76 \%$ respectively.

\section{GenBank accession numbers}

Sequences obtained in this study have been deposited in the GenBank database under the following accession numbers: MK347206-MK347212 (tick identification), MK405447-MK405477 (rickettsia omp A gene), MK405386-MK405446 (rickettsia omp B gene).

\section{DISCUSSION}

There have been a notable change in the epidemiology of rickettsial spotted fever globally and these could be attributed to warmer temperatures and increased relative humidity that have occurred in recent times due to cycles of drought and rains. These cycles stimulates increased physiological processes that in turn lead to rapid growth and ultimately accelerated reproduction. Other factors that could be responsible for the observed notable changes are;-i) infringement of humans into ecological territories that are ticks reserves as a result of increased human population, ii) increased human contact with ticks which are reservoirs and transmitters of tick-borne SFGR pathogens due to increased outdoor activities, iii) improved global trade and travels that have facilitated the distribution of tick vectors and their suitable animal hosts in a short space of time, iv) as well as migration of animals including birds which have the capacity to introduce exotic ticks and their pathogens into new territories.

Genetic materials of $R$. africae, $R$. parkeri and $R$. tamurae were the three SFGR that were detected amongst 
the ticks genera collected in this study. These three species are closely related and they are the etiologic agents of African and American tick bite fever that are very prevalent in the sub-Saharan African, United States of America and Brazil and rickettsiosis in Japan respectively (Bogovic et al., 2016). African tick bite fever is generally transmitted by Amblyomma ticks which servers as its host and wild rodents are it reservoir from which it is transmitted to it humans through ticks bite (Bogovic et al., 2016).

Even though rickettsial diseases are found globally, there is no one single tick-borne rickettsial diseases that is found all over the world rather they are restricted to a given geographical regions and are transmitted by ticks inhabiting the given region. Majority of the populace living in the sub-Saharan Africa might be seropositive to $R$. africae but hardly do they succumb to African tick bite fever as it is with travelers to the endemic regions of Africa. Sero-prevalence of $R$. africae in Cameroon is between $11.9 \%-51.8 \%$ while in Senegal it ranges between 21.4\% -51\% (Ndip et al., 2004; Mediannikov et al., 2010; Consigny et al., 2005). In a group of 940 travelers to South Africa, majority (27\%) of them had flulike symptoms as a result of contacting $R$. africae the etiologic agent of African tick-bite fever upon returning from their travel (Prabhu et al., 2011). Also Prabhu et al., (2011), reported a seroprevalence of $51.7 \%$ among inpatients identified with febrile fever who were tested for acute SFGR and TGR in Moshi, Tanzania. May June Thu ${ }^{1}$ Unit of Risk Analysis and Management, Hokkaido University Research Center for Zoonosis Control, N 20 W 10, Kita-ku, Sapporo, 001-0020 Japan

${ }^{2}$ Laboratory of Parasitology, Faculty of Veterinary Medicine, Graduate School of Infectious Diseases, Hokkaido University, N 18 W 9, Kita-ku, Sapporo, 060-0818 Japan

Find articles by May June Thu

\section{Yongjin Qiu}

${ }^{3}$ Hokudai Center for Zoonosis Control in Zambia, School of Veterinary Medicine, University of Zambia, P. O. Box 32379, Lusaka, Zambia

Find articles by Yongjin Qiu

\section{Keita Matsuno}

${ }^{4}$ Laboratory of Microbiology, Faculty of Veterinary Medicine, Graduate School of Infectious Diseases, Hokkaido University, N 18 W 9, Kita-ku, Sapporo, 060-0818 Japan

${ }^{5}$ Global Station for Zoonosis Control, Global Institution for Collaborative Research and Education (GICoRE), Hokkaido University, N 18 W 9, Kita-ku, Sapporo, 060-0818 Japan

Find articles by Keita Matsuno

\section{Masahiro Kajihara}

${ }^{6}$ Division of Global Epidemiology, Hokkaido University Research Center for Zoonosis Control, N 20 W 10, Kita-ku, Sapporo, 001-0020 Japan

Find articles by Masahiro Kajihara

\section{Akina Mori-Kajihara}

${ }^{6}$ Division of Global Epidemiology, Hokkaido University Research Center for Zoonosis Control, N 20 W 10, Kita-ku, Sapporo, 001-0020 Japan

Find articles by Akina Mori-Kajihara 


\section{Ryosuke Omori}

${ }^{7}$ Division of Bioinformatics, Hokkaido University Research Center for Zoonosis Control, N 20 W 10, Kita-ku, Sapporo, 001-0020 Japan

${ }^{8}$ Precursory Research for Embryonic Science and Technology (PRESTO), Japan Science and Technology Agency, Saitama, 332-0012 Japan

Find articles by Ryosuke Omori

\section{Naota Monma}

${ }^{9}$ Department of Infection Control, Fukushima Medical University, 1 Hikarigaoka, Fukushima, 960-1295 Japan

Find articles by Naota Monma

\section{Kazuki Chiba}

${ }^{10}$ Fukushima Institute for Public Health, 16-6 Mitouchi Houkida, Fukushima, 960-8560 Japan

Find articles by Kazuki Chiba

\section{Junji Seto}

${ }^{11}$ Yamagata Prefectural Institute of Public Health, 1-6-6 Toka-machi, Yamagata, 990-0031 Japan

Find articles by Junji Seto

\section{Mutsuyo Gokuden}

${ }^{12}$ Kagoshima Prefectural Institute for Environmental Research and Public Health, 11-40 Kinko cho, Kagoshima, 892-0835 Japan

Find articles by Mutsuyo Gokuden

\section{Masako Andoh}

${ }^{13}$ Laboratory of Veterinary Public Health, Joint Faculty of Veterinary Medicine, Kagoshima University, 1-21-24 Korimoto, Kagoshima, 890-0065 Japan

Find articles by Masako Andoh

\section{Hideo Oosako}

${ }^{14}$ Kumamoto Prefectural Institute of Public-Health and Environmental Science, Uto-shi, Kumamoto, 8690425 Japan

Find articles by Hideo Oosako

\section{Ken Katakura}

${ }^{2}$ Laboratory of Parasitology, Faculty of Veterinary Medicine, Graduate School of Infectious Diseases, Hokkaido University, N 18 W 9, Kita-ku, Sapporo, 060-0818 Japan

Find articles by Ken Katakura 


\section{Ayato Takada}

${ }^{5}$ Global Station for Zoonosis Control, Global Institution for Collaborative Research and Education (GICoRE), Hokkaido University, N 18 W 9, Kita-ku, Sapporo, 060-0818 Japan

${ }^{6}$ Division of Global Epidemiology, Hokkaido University Research Center for Zoonosis Control, N 20 W 10, Kita-ku, Sapporo, 001-0020 Japan

Find articles by Ayato Takada

\section{Chihiro Sugimoto}

${ }^{5}$ Global Station for Zoonosis Control, Global Institution for Collaborative Research and Education (GICoRE), Hokkaido University, N 18 W 9, Kita-ku, Sapporo, 060-0818 Japan

${ }^{15}$ Division of Collaboration and Education, Hokkaido University Research Center for Zoonosis Control, N 20 W 10, Kita-ku, Sapporo, 001-0020 Japan

Find articles by Chihiro Sugimoto

\section{Norikazu Isoda}

${ }^{1}$ Unit of Risk Analysis and Management, Hokkaido University Research Center for Zoonosis Control, N 20 W 10, Kita-ku, Sapporo, 001-0020 Japan

${ }^{5}$ Global Station for Zoonosis Control, Global Institution for Collaborative Research and Education (GICoRE), Hokkaido University, N 18 W 9, Kita-ku, Sapporo, 060-0818 Japan

Find articles by Norikazu Isoda

\section{Ryo Nakao}

${ }^{2}$ Laboratory of Parasitology, Faculty of Veterinary Medicine, Graduate School of Infectious Diseases, Hokkaido University, N 18 W 9, Kita-ku, Sapporo, 060-0818 Japan

Find articles by Ryo Nakao

The majority of data on ATBF cases that have been documented to date have been obtained from tourists returning from countries to which it is endemic, most from the Southern Africa countries like Botswana, South Africa, and Zimbabwe (Nilssona et al., 2017; Prebhu et al., 2011; Bogovic et al., 2016; Socolovschi et al., 2007). Bogovic et al. (2016) reported a case of ATBF in a Slovenian traveler returning from Uganda. The 29-year-old Slovenian man without underlying illnesses sought care after returning from a two weeks visit to Uganda for fever, chills, pains and complained of a tick bite a day prior to departing the country. Lorusso et al. (2013) were the first people to report about $R$. africae in ticks in Uganda where R. conorii had previously been reported by Socolovschi et al. (2007) as being prevalent. Similarly, Angerami et al. (2018) reported ATBF on a Brazilian who visited South Africa upon his return to Brazil. He had eschar and symptoms characteristics of ATBF which was confirmed by both immunological and molecular diagnostic methods to be infected by $R$. africae. African tick bite fever had also been reported for the first time by Harrison et al. (2016) on an Austrian traveler to East Africa who acquired the disease through tick bite during a visit to Tanzania. ATBF is generally a mild disease and to date, there has not been any reported deaths attributed to infection of $R$. africae.However, just like $R$. parkeri rickettsiosis, the disease caused by $R$. africae is often associated with an inoculation eschar at the spot of attachment of the tick vector. Usually, the symptoms associated with ATBF normally appear many days after the development of the eschar and they are usually that of fever, headache, myalgia, regional lymphadenopathy and generalized rash in about $50 \%$ of the cases.

Amblyomma variegatum tick has been reported to be the vector of $R$. africae with a prevalence of $97.1 \%$ in Uganda (Nakao et al., 2013). However, Waner et al. (2014), reported finding the DNA ofR. africae 
in Hyalomma detritum tick collected from a wild boar in Israel indicating that the spotted fever group rickettsia is not limited in distribution to the African continent nor to a given host tick. Similarly, Yssouf et al. (2014) reported the detection of $R$. africae in $90 \%$ of $A$. variegatum , $1 \%$ of $R$. appendiculatus and $2.7 \%$ of Rhipicephalus (Boophilus) microplusin study ticks collected from locally domesticated animals in the Union of the Comoros, as well as in $77.14 \%$ in A. variegatum ticks obtained from cattle imported into the country. Also, Maina et al. (2014) reported the detection of $R$. africae-genotype DNA in $92.6 \%$ of adult $A$. variegatum ticks collected from domestic ruminants in Kenya even though they found no evidence of the pathogen in blood specimens in the domestic animals sampled. $R$. africaegenetic materials have been detected by PCR from different species of ticks belonging to Amblyomma, Rhipicephalus, Hyalomma genera in several African countries such as Mali, Senegal, Guinea, Liberia, Sudan, Democratic Republic of Congo, Cameroon, Nigeria, Niger, Kenya and Burundi (Parola et al., 2013; Bogovic et al., 2016) and these reports are in consonant with our finding as the DNA of $R$. africae was detected in the different genera of ticks that we assessed.

R. parkeri a member of the spotted fever group rickettsia is the etiologic agent of American tick bite fever that is prevalent in the South and North America continents is transmitted by Amblyomma species. The spotted fever disease associated with the organism is characterized by eschar related ailments in humans which are similar to symptoms of Rocky Mountain spotted fever. The index rickettsiosis spotted fever case caused by $R$. parkeri was first recognized by Paddock et al., (2004) and ever since then; numerous cases have been identified and reported in many southeastern states of the USA (Kimita et al., 2016; Paddock et al., 2008). Cowdry, (1993), was the first to describe the finding of the organism in the tissues and eggs of female A. maculatum ticks that were collected in Jackson County, Missouri. However, Parker et al. (1963), isolated the organism for the first time from Gulf Coast ticks that were collected in South eastern Texas and ever since then, $R$. parkeri a SFG rickettsiae have been frequently detected in A. maculatum. However, R. parkerihas been detected in other tick species other than A. maculatumas Williamson et al., (2010), reported the detection of its DNA inD. variabilis in ticks removed from persons in Texas, USA.R. parkeri infections in dogs and cows have been described in southeastern United States. Infection of humans by $R$. parkeri in most cases is associated with a necrotic eschar at the point of inoculation after several days of an infected tick bite and it is usually with a low grade to moderate fever that is very similar to RMSF though less in severity. Some of the symptoms associated with $R$. parkeri rickettsioses are fever, inoculation eschar, macules or papules rashes, vesicles or pustules, petechiae on palms or soles, headache, myalgias, sore throat, lymphadenopathy, diarrhea, nausea or vomiting (Paddock et al., 2008). No case of R.parkeri rickettsiosis have however been reported in Central America though A. maculatum is widely distributed throughout the region even though a mild eschar-related rickettsiosis that is very akin to $R$. parkeririckettsiosis have been reported in a traveler who returned from Honduras (Paddock et al., 2008). Since the first human disease case caused by $R$. parkeri was reported by Paddock et al. (2004), numerous cases of rickettsioses caused by $R$. parkeri have been reported among persons residing in the ecological range of the vector tick $A$. maculatum in the USA. Infections and eschar associated illness with $R$. parkeri have been frequently reported in several Latin American countries such as Argentina, Brazil, and Uruguay, and the organism has been detected in A. tristeticks (Romer et al., 2011). For the first time, here we report the detection of genetic material of $R$. parkeri in the African continent and the epidemiological implications are not well known. However, because it has been documented as a human pathogen, its involvement in human cases in the study sites may not be unlikely as it may probably have gone undetected. We observed discordant phylogenetic assignments of the omp A and omp B genes of sample 188 as they were found to cluster with Candidatus_Rickettsia EU27216.1 and $R$. parkeri KY113110 respectively in Figs 4 and 5 and this was shown to be so with nucleotide and amino acid alignments as shown in Figures $2 \mathrm{~A}$ to D.

Rickettsia sp. strain Ga-Seema is an incompletely described rickettsial that was detected from three fed adult maleRhipicephalus simus ticks collected from two donkeys in 2014 in Hlahlagane, Limpopo Province, South Africa by Halajian et al. (2018) which has not been reported previously and its pathogenic potential is currently unknown.

R. tamurae infection according to Imaokaa et al. (2013) is associated with symptoms such as mild local 
inflammatory signs like swellings, erythema, redness heat and pain. Symptoms of $R$. tamurae infection mimics cellulitis with increased serum titers of antibody against the organism (Halajian et al., 2018). Unlike most SFGR, infection with R.tamurae is not associated with high fever, generalized rash, lymphadenopathy as it is often seen in other spotted fever rickettsioses. R.tamurae was first isolated from A. testudinarium ticks in Japan and has the wild boar and domestic pigs as it primary host although it can also infest deer, cattle, other ungulates and domestic livestock as well as humans (Halajian et al., 2018; Imaokaa et al., 2011; Motoi et al., 2013). R. tamurae has been isolated from the skin biopsy specimen from wild boars and also in ticks (Halajian et al., 2018). It was previously thought to be non-pathogenic to humans until it was reported in human cases in Japan (Halajian et al., 2018) as well as in Laos where its involvement in spotted fever case was documented after a patient tested seropositive for the organism (Gaowa et al., 2013). Phylogenetic analyses of the omp A and omp B sequences of sample 209 assigned them as $R$. africae and $R$. tamurae respectively and homology search confirmed that omp A sequence is $R$. africae while theomp $\mathrm{B}$ sequence had $100 \%$ similarity with $R$. tamurae. We performed nucleotide and amino acid sequences alignments with the two sequences as shown in Figures 3A-D, the omp B showed complete homology with $R$. tamurae indicating that sample B209 is most highly $R$. tamurae in the omp B gene region while theomp A was closely related with $R$. africae. We are not sure if recombination did occur in the two genes in question. Further study like full genome sequencing is needed to elucidate this observation. $R$. tamurae has been associated with different Amblyommaspp. as reported by Blanco (Blanco et al.,2017) who detected the pathogen in screened nymphs of $A$. ovale tick collected from small mammals such as wild rodents and marsupials in Brazil while a recent report stated its detection in a Haemaphysalis megaspinosa tick (Blanco et al., 2017). However, this is the first report of $R$. tamurae- like pathogen, the agent of SFG rickettsiosis in Japan and some Far East Asian countries in A. variegatum tick collected from cattle in the African continent.

Ticks as well as the diseases they transmit have an ecological range constrained by animal host diversity, movement and climatic factors. The current rapid expansion of ticks and the diseases they transmit into new ecological niches can be attributed to increased mobility of pets and animal migration over long distances. Due to climate change, new and favorable niches are being created thus making the spread of ticks very rapid over a wide range of ecological zones (Nooroong et al., 2018). These might be possible factors why there is an increasing spread of ticks and tick-borne diseases globally. For example, in Germany,Dermacentor reticulatus has spread to over a large part of the country along with babesiosis that they transmit (Phongmany et al., 2006) just as the ecological range of Ixodes ricinus the agent that transmit anaplasmosis and Lyme borreliosis has extended greatly in Sweden of recent (Gaowa et al., 2013). Similarly, D. veriabilisthe host and vector of RMSF has spread to the North-East of United States of America (Berglund et al., 1995). The current climate change has been reported to be the reason for these observable spread of ticks and their vectored pathogens. With the change in global climate, increased interaction of humans with ticks and expansive global trade in animals as well as the migratory nature of animals (Heile et al., 2006; Berglund et al., 1995; CDC, 2019; Palomar et al., 2012; Sparagano et al., 2015), it is not unlikely that ticks-borne pathogens could be easily introduced into new ecological niches thus fueling the global spread of tick-borne diseases.

\section{Conclusion:}

We have reported on the detection of tick-borne rickettsia pathogens which are the etiologic agents of spotted fever group rickettsioses. We assessed ticks collected from domestic animals and found the DNA of $R$. africae, $R$. parkeri and $R$. tamurae in the three genera of ticks collected in this study. The detection of genetic materials of $R$. parkeri and $R$. tamurae in ticks collected in Africa calls for further studies to elucidate this result. The discordance of the $о m p \mathrm{~A}$ and $о m p \mathrm{~B}$ genes fragments of the same samples is also another observation that should be studied further in detail. Full genome sequencing or a complete omp A and $o m p$ B would have helped to further resolve these controversies but insufficient resources constrained us. Usually, the SFGR are not considered in the diagnosis and treatment of patients with signs and symptoms of fever in these rural communities. This data is therefore a wakeup call on health personal in the study communities to consider infections caused by these pathogens as they may probably be responsible for the flulike symptoms presented by their patients. They should also educate the community member on the health implications of ticks' bites and the need to report such bites as quickly as possible. Aggressive ticks 
control strategies should be commenced by the relevant government agencies so as to mitigate the health implications of ticks' infestations. Further studies are needed to demonstrate the competence of $A$. hebraeum as regarding acquisition and transmission of $R$. parkeri as well as changes in the endosymbionts in the tick species. The limitations of the study are that tick genders and feeding states were not considered however, these do not in any way diminish the relevance of the findings of the study.

\section{Acknowledgments}

We thank the farmers who allowed us access into the farms for sample collection and for their assistance in sample collection.

\section{E TH I C A L A PPROVA L}

Ethical clearance certificate (cert number; OBI013) was obtained from University of Fort Hare research and ethics committee (UREC).

\section{CO N FLI C T O F I NTERE S T}

All authors declare no conflict of interest.

\section{AUTH O R S CO NTRI B UTI O N S}

The study was designed by BCI. The sampling, laboratory work and bioinformatics analyses and writeup were carried out by BCI, AN, while BCI, LCO and AIO supervised the study and contributed to the interpretation of the data, manuscript editing and approval of the final manuscript.

Data Availability Statement: The data that support the findings of this study are openly available in [GenBank] at https: //www.ncbi.nlm.nih.gov/nuccore, MK347206-MK347212 (tick identification), MK405447-MK405477 (rickettsia omp A gene), and MK405386-MK405446 (rickettsia omp B gene).

\section{ORCID}

Benson Chuks Iweriebor https://orcid.Org/0000-0002-5269-1849

\section{References}

1. Parola, P., Paddock, C.D., Socolovschi, C., Labruna, M.B., Mediannikov, O., Kernif, T. et al. (2013). Update on Tick-Borne Rickettsioses around the World: a Geographic Approach. Clinical Microbiology Review, p.657-702. doi:10.1128/CMR.00032-13 PMCID: PMC3811236.

2. Raoult, D., Roux, V. (1997). Rickettsioses as paradigms of new or emerging infectious diseases. Clinical Microbiology Review,10:694-719.

3. Bogovic, P., Lotric-Furlan, S., Korva, M., Avsic-Zupanc, T. (2016). African Tick-Bite Fever in Traveler Returning to Slovenia from Uganda.Emerging Infectious Diseases , 22 (10). DOI: http://dx.doi.org/10.3201/eid2210.160650

4. Portillo, A., de Sousa, R., Santibáñez, S., Duarte, A., Edouard, S., IFonseca, I.P, Marques, C., Novakova, M., Palomar, A.M., Santos, M. (2017). Guidelines for the detection of Rickettsia spp. Vector Borne Zoonotic Diseases, 17:23-32.

5. Socolovschi, C., Mediannikov, O., Raoult, D., Parola, P. (2009). The relationship between spotted fever group Rickettsiae and ixodid ticks. Veterinary Research , 40:34

6. Tomassone, L., Portillo, A., Nováková, M., Sousa, R., Oteo, J.A. (2018). Neglected aspects of tick-borne rickettsioses. Parasites 83 Vectors, 11:263 https://doi.org/10.1186/s13071-018-2856-y

7. Parola, P., Paddock, C.D., Raoult, D. (2005). Tick-borne rickettsioses around the world: emerging diseases challenging old concepts. Clinical Microbiology Review 18:719-756.

8. Iweriebor, B.C., Mmbaga, E.J., Adegborioye, A., Igwaran, A., Obi, L.C., Okoh, A.I. (2017). Genetic profiling for Anaplasma and Ehrlichia species in ticks collected in the Eastern Cape Province of South Africa. BMC microbiology, 17(1), p.45.

9. Yawa, M., Nyangiwe, N., Kadzere, C.T., Muchenje, V., Mpendulo, T.C., Marufu, M.C. (2019). In search of the Rhipicephalus (Boophilus) microplus in the western-central regions of the 
Eastern Cape Province, South Africa. Ticks and Tick-borne Diseases , 10(3) 564-567. https://doi.org/10.1016/j.ttbdis.2019.01.009

10. Walker, A.R., Bouattour, A., Camicas, J.L., Estrada-Pana, A., Horak, I.G., Latif, A.A. (2003). Ticks of Domestic Animals in Africa; A Guide to Identification of Species. Bioscience Reports , University of Edinburgh ISBN 0-9545173-0-X

11. Williamson, P.C., Billingsley, P.M., Teltow, G.J., Seals, J.P., Turnbough, M.A., Atkinson, S.F. (2010). Borrelia, Ehrlichia, and Rickettsia spp. in Ticks Removed from Persons, Texas, USA.Emerging Infectious Diseases, 16, 3, DOI: 10.3201/eid1603.091333

12. Regnery, R.L., Spruill, C.L. and Plikaytis, B.D. (1991). Genotypic identification of rickettsiae and estimation of intraspecies sequence divergence for portions of two rickettsial genes. Journal of bacteriology , 1991; 173(5), pp.1576-1589.

13. Eremeeva, M., Yu, X., Raoult, D. (1994). Differentiation among spotted fever group rickettsiae species by analysis of restriction fragment length polymorphism of PCR-amplified DNA. Journal of Clinical Microbiology , 32:803-10

14. Kollars, T.M Jr, Kengluecha A. (2001). Spotted fever groupRickettsia in Dermacentor variabilis (Acari: Ixodidae) infesting raccoons (Carnivora:Procyonidae) and opossums (Marsupialia: Didelphimorphidae) in Tennessee. Journal of Medical Entomology,38:601-2. DOI: 10.1603/0022-2585-38.4.601

15. Altschul, S.F., Gish. W., Miller, W., Myers, E.W., Lipman, D.J. (1990). Basic local alignment search tool. Journal of Molecular Biology, 215(3), pp.403-410.

16. Saitou, N., Nei, M. (1987). The neighbor-joining method: A new method for reconstructing phylogenetic trees. Molecular Biology and Evolution, 4:406-425.

17. Felsenstein, J. (1985). Confidence limits on phylogenies: An approach using the bootstrap. Evolution, 39:783-791.

18. Nei, M., and Kumar, S. (2000). Molecular Evolution and Phylogenetics. Oxford University Press, New York.

19. Kumar, S., Stecher, G., and Tamura, K. (2016). MEGA7: Molecular Evolutionary Genetics Analysis version 7.0 for bigger datasets. Molecular Biology and Evolution, 33:1870-1874

20. Ndip, L.M., Fokam, E.B., Bouyer, D.H., Ndip, R.N., Titanji, V.P., Walker, D.H. (2004). Detection of Rickettsia africae inpatients and ticks along the coastal region of Cameroon. American Journal of Tropical Medicine and Hygiene, 71:363-6.

21. Mediannikov, O., Diatta, G., Fenollar, F., Sokhna, C., Trape, J.F., Raoult, D. (2010). Tickborne rickettsioses, neglected emerging diseases in rural Senegal. PLoS Neglected Tropical Diseases,14;4(9).pii:e821.doi:10. 1371/journal.pntd.0000821PMID:20856858

22. Consigny, P.H., Rolain. J., Mizzi, D., Raoult. D. (2005). African Tick-bite Fever in French Travelers. Emerging Infectious Diseases, ${ }^{*}$ www.cdc.gov/eid ${ }^{*}$ Vol. 11, No. 11,

23. Prabhu, M., Nicholson, W.L., Roche, A.J., Kersh, G.J., Fitzpatrick, K.A., Oliver, L.D., Massung, R.F., et al. (2011). Q Fever, Spotted Fever Group, and Typhus Group Rickettsioses Among Hospitalized Febrile Patients in Northern Tanzania. Clinical Infectious Diseases , 53(4): e8-e15. doi: 10.1093/cid/cir411 PMCID: PMC3148261

24. Jensenius, M., Fournier, P.E., Vene, S., Hoel, T., Hasle, G., Henriksen, A.Z., et al. (2003). Norwegian African Tick Bite Fever Study Group. African tick bite fever in travelers to rural sub-Equatorial Africa. Clinical Infectious Diseases,36:1411-7. http://dx.doi.org/10.1086/375083

25. Nilssona, K., Wallmeniusa, K., Rundlof-Nygrenb, P., Stromdah, S., Pahlso, C. (2017). African tick bite fever in returning Swedish travelers. Report of two cases and aspects of diagnostics INFECT ECO EPIDEM 7, 1343081 doi.org/10.1080/20008686.2017.1343081

26. Lorusso, V., Gruszka, K.A., Majekodunmi, A., Igweh, A., Welburn, S.C., Picozzi, K. (2013). Rickettsia africae in Amblyomma variegatum ticks, Uganda and Nigeria. Emerging Infectious Diseases , 19:17057.10.3201/eid1910.130389

27. Socolovschi, C., Matsumoto, K., Marie, J.L., Davoust, B., Raoult, D., Parola, P. (2007). Identification of rickettsiae, Uganda and Djibouti.Emerging Infectious Diseases, 13:1508-10. doi.org/10.3201/ eid1310.070078 
28. Angerami, R.N., Krawczak, F.S., Nieri-Bastos, F.A, Santos, F., Medorima, C., Resende, M.R. (2018). First report of African tick-bite fever in a South American traveler. SAGE Open Med Case Reports , 6(1 -3) DOI: 10.1177/2050313X18775301

29. Harrison, N., Burgmann, H., Forstner, C., Ramharter, M., Szell, M., Schotta, A. (2016). Molecular diagnosis of African tick bite fever using eschar swabs in a traveler returning from Tanzania. Wien Klin Wochenschr, 128:602-605 DOI 10.1007/s00508-016-1047-0

30. Nakao, R., Qiu, Y., Igarashi, M., Magona, J.W., Zhou, L., Ito, K., Sugimoto, C. (2013). High prevalence of spotted fever group rickettsiae in Amblyomma variegatum from Uganda and their identification using sizes of intergenic spacers. Ticks and Tick-Borne Diseases , 4:506-12. http://dx.doi.org/10.1016/j.ttbdis.2013.07.001

31. Waner, T., Keysary, A., Eremeeva, M.E., Din, A.B., Mumcuoglu, K.Y., King, R., Atiya-Nasagi, Y. (2014). Rickettsia africae and Candidatus Rickettsia barbariae in Ticks in Israel. The American Society of Tropical Medicine and Hygiene, 90(5), pp. 920-922 doi:10.4269/ajtmh.13-0697.

32. Yssouf, A., Socolovschi, C., Kernif, T., Temmam, S., ELagadec, E., Tortosa, P., Parola, P. et al. (2014). First molecular detection of Rickettsia africae in ticks from the Union of the Comoros.Parasites \&6 Vectors , 7:444 http://www.parasitesandvectors.com/content/7/1/444

33. Maina, A.N., Jiang, J., Omulo, S.A., Cutler, S.J., Ade, F., Ogola, E., Feikin, D.R., Njenga, M.K., Cleaveland, S., Mpoke, S., et al, (2014). High Prevalence of Rickettsia africae Variants in Amblyomma variegatum Ticks from Domestic Mammals in Rural Western Kenya: Implications for Human Health. Vector-Borne Zoonotic Diseases, 14 (10), DOI: 10.1089/vbz.2014.1578

34. Ehounoud, C.B., Yao, K.P., Dahmani, M., Achi, Y.L., Amanzougaghene, N., N'Douba, A.K., N'Guessan, J.D., Raoult, D., Fenollar, F., Mediannikov, O. (2016). Multiple Pathogens Including Potential New Species in Tick Vectors in Cote d'Ivoire. PLOS Neglected Tropical Diseases, |DOI:10.1371/journal.pntd.0004367

35. Kimita, G., Mutai, B., Nyanjom, S.G., Wamunyokoli, F., Waitumbi, J. (2016). Phylogenetic Variants of Rickettsia africae, and Incidental Identification of "Candidatus Rickettsia Moyalensis" in Kenya.PLOS Neglected Tropical Diseases, DOI:10.1371/0004788

36. Paddock, C.D., Sumner, J.W., Comer, J.A., Zaki, S.R., Goldsmith, C.S., Goddard, J., McLellan, S.L., Tamminga, C.L., Ohl, C.A. (2004). Rickettsia parkeri: a newly recognized cause of spotted fever rickettsiosis in the United States. Clinical Infectious Diseases, 15;38(6):805-11.

37. Paddock, C.D., Finley, R.W., Wright, C.S., Robinson, H.N., Schrodt, B.J., Lane, C.C., Ekenna, O., Blass, M.A., Tamminga, C.L., Ohl, C.A. et al. (2008). Rickettsia parkeri rickettsiosis and its clinical distinction from Rocky Mountain spotted fever. Clinical Infectious Diseases, 47: 1188-1196.

38. Whitman, T.J., Richards, A.L., Paddock, C.D., Tamminga, C.L., Sniezek, P.J., Jiang, J., Byers, D.K., Sanders, J.W. (2007). Rickettsia parkeri infection after tick bite, Virginia.Emerging Infectious Diseases, 13:334-336. 58.

39. Cowdry, E.V. (1993). The distribution of rickettsia in the tissues of insects and arachnids. Journal of Experimental Medicine , 37:431-459

40. Parker, R.R., Kohls, G.M., Cox, G.W., Davis, G.E. (1939). Observations on an infectious agent from Amblyomma maculatum. Public Health Report, 139; 54:1482-1484.

41. Romer, Y., Seijo, A.C., Crudo, F., Nicholson, W.L., Varela-Stokes, A., Lash, R.R., et al. (2011). Rickettsia parkeri Rickettsiosis, Argentina.Emerging Infectious Diseases , 17(7): 1169-1173. doi: 10.3201/eid1707.101857

42. Chen, L.H., Wilson, M.E. (2009). Tick-Borne Rickettsiosis in Traveler Returning from Honduras.Emerging Infectious Diseases, 15(8): 1321-1323.doi: 10.3201/eid1508.090172

43. Halajian, A., Palomar, A.M., Portillo, A., Heyne, H., Romero, L., Oteo, J.A. (2018). Detection of zoonotic agents and a new Rickettsia strain in ticks from donkeys from South Africa: Implications for travel medicine. Travel Medicine and Infectious Diseases , 26:43-50. doi: 10.1016/j.tmaid.2018.10.007

44. Imaokaa, K., Kanekoa, S., Tabarab, K., Kusatakea, K., Morita, E. (2011). The First Human Case of Rickettsia tamurae Infection in Japan. Case Report in Dermatology, 3:68-73 DOI: 10.1159/000326941

45. Motoi, Y., Asano, M., Inokuma, H., Ando, S., Kawabata, H., Takano, A., Suzuki, M. (2013). Detection 
of Rickettsia tamurae DNA in ticks and wild boar (Sus scrofa leucomystax) skins in Shimane Prefecture, Japan. Journal of Veterinary Medical Science, 75:263-267.

46. Gaowa, N.O., Aochi, M., Wuritu, Wu. D., Yoshikawa, Y., Kawamori, F., Honda, T., Fujita, H., Takada, N., Oikawa, Y. et al. (2013). Rickettsiae in Ticks, Japan, 2007-2011.Emerging Infectious Diseases, 19(2): 338-340. doi: 10.3201/eid1902.120856 PMID: 23460996

47. Thu, M.J., Qiu, Y., Matsuno, K., Kajihara, M., Mori-Kajihara, A., Omori, R., Monma, N., Chiba, K., Seto, J., Gokuden, M. et al. (2019). Diversity of spotted fever group rickettsiae and their association with host ticks in Japan.Science Report, 9: 1500. PMID: 30728409

48. Blanco, C.M., Teixeira, B.R., da Silva, A.G., de Oliveira, R.C., Strecht, L., Ogrzewalska, M., de Lemos, E.R.S. (2017). Microorganisms in ticks (Acari: Ixodidae) collected on marsupials and rodents from Santa Catarina, Parana and Mato Grosso do Sul states, Brazil. Ticks and tick-borne Diseases, 8: (1), pp.90-98.

49. Nooroong, P., Trinachartvanit, W., Baimai, V., Ahantarig, A. (2018). Phylogenetic studies of bacteria (Rickettsia, Coxiella, and Anaplasma) in Amblyomma and Dermacentor ticks in Thailand and their co-infection. Ticks and tick-borne Diseases, 9:(4), pp.963-971.

50. Phongmany, S., Rolain, J.M., Phetsouvanh, R., Blacksell, S.D., Soukkhaseum, V., Rasachack, B., Phiasakha, K., Soukkhaseum, .S, Frichithavong, K., Chu, V., et al. (2006). Rickettsial infections and fever, Vientiane, Laos.Emerging Infectious Diseases, 12:256-262.

51. Medlock, J.M., Hansford, K.M., Bormane, A., Derdakova, M., Estrada-Pena, A., George, J.C., Golovljova, I., Jaenson, T.G., Jensen, J.K., Jensen, P.M,. et al. (2013). Driving forces for changes in geographical distribution of Ixodes ricinus ticks in Europe. Parasitology and Vectors, 6:1

52. Heile, C., Heydorn, A.O., Schein, E. (2006). Dermacentor reticulatus (Fabricius, 1794) - Distribution, biology and vector for Babesia canis in Germany. Berliner und Munchener tierarztliche Wochenschrift , 119(7-8):330-4

53. Berglund, J., Eitrem, R., Ornstein, K., Lindberg, A., Ringer, A., Elmrud, H., Carlsson, M., Runehagen, A., Svanborg, C., Norrby, R. (1995). An Epidemiologic Study of Lyme disease in Southern Sweden. New England Journal of Medicine, 333:1319-24

54. CDC. (2019). Rocky Mountain spotted fever (RMSF). Content source: Centers for Disease Control and Prevention, National Center for Emerging and Zoonotic Infectious Diseases (NCEZID), Division of Vector-Borne Diseases, (DVBD).

55. Palomar, A.M., Santibanez, P., Mazuelas, D., Roncero, L., Santibanez, S., Portillo, A., Oteo, J.A. (2012). Role of birds in dispersal of etiologic agents of tick-borne zoonoses, Spain, 2009. Emerging Infectious Diseases , 18:1188-91. 80.

56. Sparagano, O., George, D., Giangaspero, A., Špitalská, E. (2015). Arthropods and associated arthropod-borne diseases transmitted by migrating birds. The case of ticks and tick-borne pathogens. Veterinary Parasitology, 213:61-6.GenBank Accession numbers of Rickettsia reference sequences used in phylogenetic analyses of the ompA and ompB genes

\section{OMPB}

DQ113910_R.tamurae, CP003341_R.parkeri, AF123706_R.africae，KX227791_R.africae, AF149110_R.conorii, KX227788_R.africae, KY113111_R.parkeri, LN794217_R. monacen-

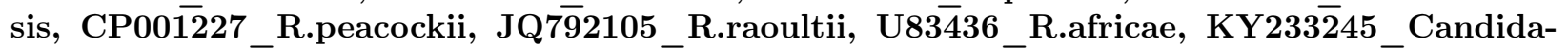
tus Rickettsia, AF123714_R. massiliae, AF123719_R. rhipicephali, HM050273_R. sibirica, KU645284_Candidatus Rickettsia, KT633262_R.africae,DQ097083_R. mongolotimonae, KX227791_R. africae, KT633262.1 R.africae, KY113111_R_parkeri , ĀF123706_R. africae, AF123717_ R. parkeri

OmpA

CP001612_R. africae, AH015610_R. raoultii, AY319290_R. rickettsia, KF702333_Candidatus Rickettsia, KY780025_R. sibirica, EU272186_Candidatus Rickettsia, MF511254_-

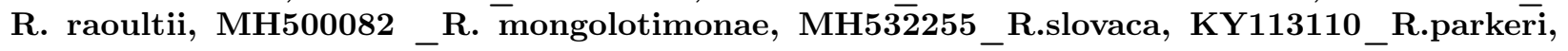


KY233245_CANDIDATUS Rickettsia barbariae, MF379309_R. sibirica, KY513920_R. sibirica, EŪ622980_R.africae, MH532254_R.slovaca, MG515014_R. africae GU247115_R. africae, KJ645933_R. africae, U43790_R. africae, DQ103259_ $\bar{R}$. tamurae, LC388791_R. tamurae,KC003476_R. parkeri,KU744412__R. parkeri

Figure 1: Neighbour-joining rectangular cladogram of $12 \mathrm{~S}$ mitochondrial rDNA of tick species generated from the study with the reference sequences from GenBank. The cladogram was constructed by using the neighbour-joining method in ClustalX 2.1 program. Test sequences intermingled with sequences of Amblyomma, Hyalomma, and Rhipicephalus species reference strains obtained from GenBank. All sequenced ticks samples clustered with the three identified genera; Amblyomma, Hyalomma and Ripicephalus reference sequences obtained from GenBank. Test sequences are in bracket and arrowed while the reference sequences are in GenBank accession number.

Figure 2a: Nucleotide sequence alignment of the ompB gene of sample B188 against the homologous reference sequence of $R$. parkeri (KY113111) indicating 100\% homology. Reference sequence was obtained based on highest percentage homology that the test sequence has with $R$. parkeri which was obtained through Nucleotide BLAST tool in the GenBank. The dots represent nucleotide similarity of the query sequence with the reference strain.

Figure 2b: Amino acid sequence alignment of the ompB gene of sample B188 against the homologous reference sequence of $R$. parkeri (KY113111) indicating 100\% homology. Reference sequence was obtained based on highest percentage homology that the test sequence has with $R$. parkeri which was obtained through Nucleotide BLAST tool in the GenBank. The dots represent amino acid similarity of the query sequence with the reference strain.

Figure 2c: Nucleotide sequence alignment of the ompA gene of sample A188 against the homologous reference sequence ofRickettsia sp. strain Ga-Seema (MG953288) indicating 95\% homology. Reference sequence was obtained based on highest percentage homology that the test sequence has with Rickettsiasp. strain Ga-Seema which was obtained through Nucleotide BLAST tool in the GenBank. The dots represent nucleotide similarity of the query sequence with the reference strain.

Figure 2d: Amino acid alignment of the ompA gene of sample A188 against the homologous reference sequence of Rickettsia sp. strain Ga-Seema (MG953288) indicating 95\% homology. Reference sequence was obtained based on highest percentage homology that the test sequence has with Rickettsia sp. strain Ga-Seema which was obtained through Nucleotide BLAST tool in the GenBank. The dots represent amino acid similarity of the query sequence with the reference strain.

Figure 3a: Nucleotide sequence alignment of the ompB gene of sample B209 against the homologous reference sequence of $R$. tamurae (DQ113910) indicating 100\% homology. Reference sequence was obtained based on highest percentage homology that the test sequence has with $R$. timurae which was obtained through Nucleotide BLAST tool in the GenBank. The dots represent nucleotide similarity of the query sequence with the reference strain.

Figure 3b: Amino acid sequence alignment of the ompB gene of sample B209 against the homologous reference sequence of $R$. tamurae (DQ113910) indicating 100\% homology. Reference sequence was obtained based on highest percentage homology that the test sequence has with $R$. timurae which was obtained through Nucleotide BLAST tool in the GenBank. The dots represent amino acid similarity of the query sequence with the reference strain.

Figure 3c: Nucleotide sequence alignment of the ompA gene of sample A209 against the homologous reference sequences of $R$. tamurae (DQ103259) and $R$. africae (EU622980) indicating $100 \%$ identity with $R$. africae whereas the omp B gene of the same sample has $100 \%$ identity with $R$. tamurae. Reference sequence was obtained based on highest percentage homology that the test sequence has with $R$. africae which was obtained through Nucleotide BLAST tool in the GenBank. The three nucleotides insert that differentiate $R$. africae and $R$. tamurae are underlined at position 97 to 99 . The 
dots represent nucleotide similarity of the query sequence with the reference strain.

Figure 3d: Amino acid sequence alignment of the ompA gene of sample A209 against the homologous reference sequences of $R$. tamurae (DQ103259) and $R$. africae (EU622980) indicating $100 \%$ identity with $R$. africae whereas the ompB gene of the same sample has $100 \%$ identity with $\boldsymbol{R}$. tamurae. Reference sequence was obtained based on highest percentage homology that the test sequence has with $R$. africae which was obtained through Nucleotide BLAST tool in the GenBank. The three nucleotides insert that differentiate $R$. africae and $R$. tamurae are underlined at position 97 to 99 . The dots represent amino acid similarity of the query sequence with the reference strain.

Figure 4: Phylogenetic tree of ompA gene sequences in bold generated from the study with the related reference sequences obtained from NCBI GenBank. The evolutionary history was inferred using the Neighbor-Joining method (Saitou and Nei, 1987). The optimal tree with the sum of branch length $=1.67432413$ is shown. The percentage of replicate trees in which the associated taxa clustered together in the bootstrap test (1000 replicates) is shown next to the branches (Felsenstein, 1985). The evolutionary distances were computed using the p-distance method (Nei and Kumar, 2000) and are in the units of the number of base differences per site. The analysis involved 67 nucleotide sequences. Codon positions included were $1 \mathrm{st}+2 \mathrm{nd}+3 \mathrm{rd}+$ Noncoding. All positions containing gaps and missing data were eliminated. There were a total of 166 positions in the final dataset. Evolutionary analyses were conducted in MEGA7 (Kumar et al., 2016). The test sequences denoted diamond red clustered with other Rickettsia references.

Figure 5: Phylogenetic tree of $o m p B$ gene sequences in bold generated from the study with the related reference sequences obtained from NCBI GenBank. The evolutionary history was inferred using the Neighbor-Joining method (Saitou and Nei, 1987). The optimal tree with the sum of branch length $=$ 2.89828155 is shown. The percentage of replicate trees in which the associated taxa clustered together in the bootstrap test (1000 replicates) is shown next to the branches (Felsenstein, 1985). The evolutionary distances were computed using the p-distance method ${ }^{18}$ and are in the units of the number of base differences per site. The analysis involved 86 nucleotide sequences. Codon positions included were 1 st $+2 \mathrm{nd}+3 \mathrm{rd}+$ Noncoding. All positions containing gaps and missing data were eliminated. There were a total of 236 positions in the final dataset. Evolutionary analyses were conducted in MEGA7 (Nei and Kumar, 2000). All study sequences clustered phylogenetically with $R$. africae sequences from GenBank with the exception of sequences B188 and B209; B188 clustered with $R$. parkeri (AF123717) while sample B209 clustered with $R$. tamurae(DQ113910) with high bootstrap values above $99 \%$. Test sequences are in bold in green dot. 


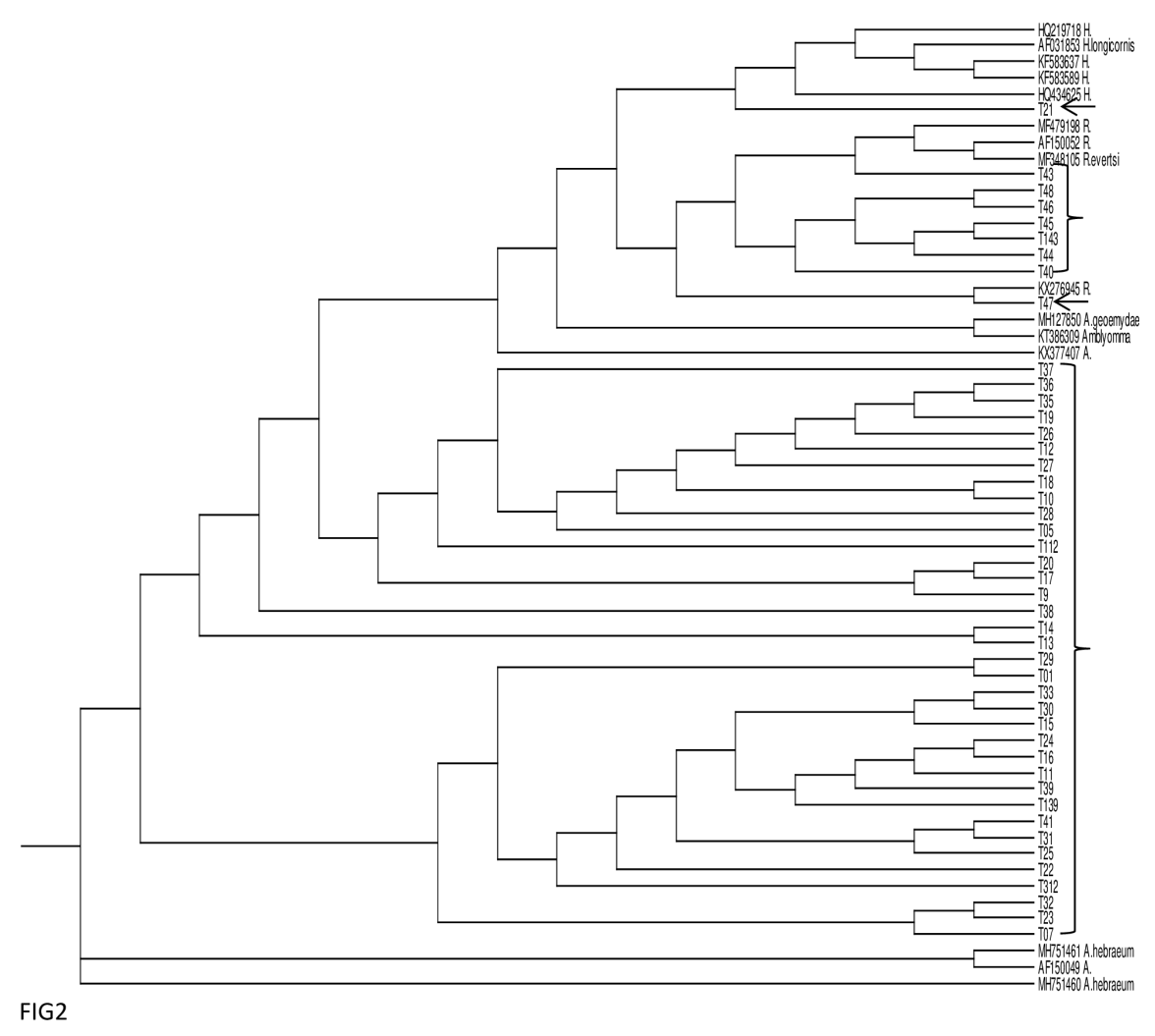

\section{Hosted file}

FIG 2A - D of AI88 NUCLEOTIDE AND AMINO ACID ALIGNMENTS.docx available at https://authorea. com/users/319008/articles/448809-genetic-evidence-of-spotted-fever-group-rickettsiae-inticks-collected-from-domestic-animals-in-eastern-cape-south-africa

\section{Hosted file}

FIG 3a, b c d- B209 and REF R.tamurae.docx available at https://authorea.com/users/319008/ articles/448809-genetic-evidence-of-spotted-fever-group-rickettsiae-in-ticks-collectedfrom-domestic-animals-in-eastern-cape-south-africa 


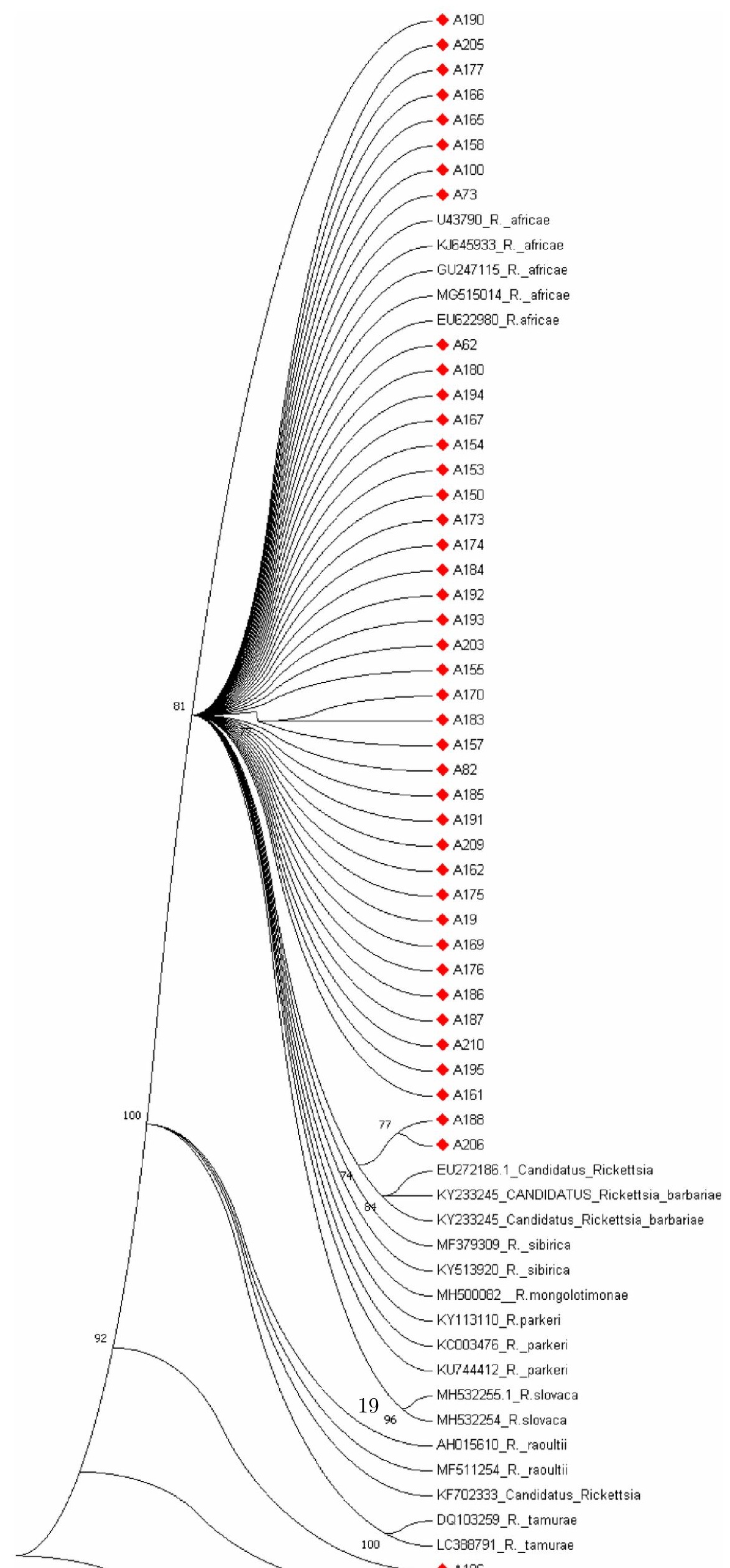




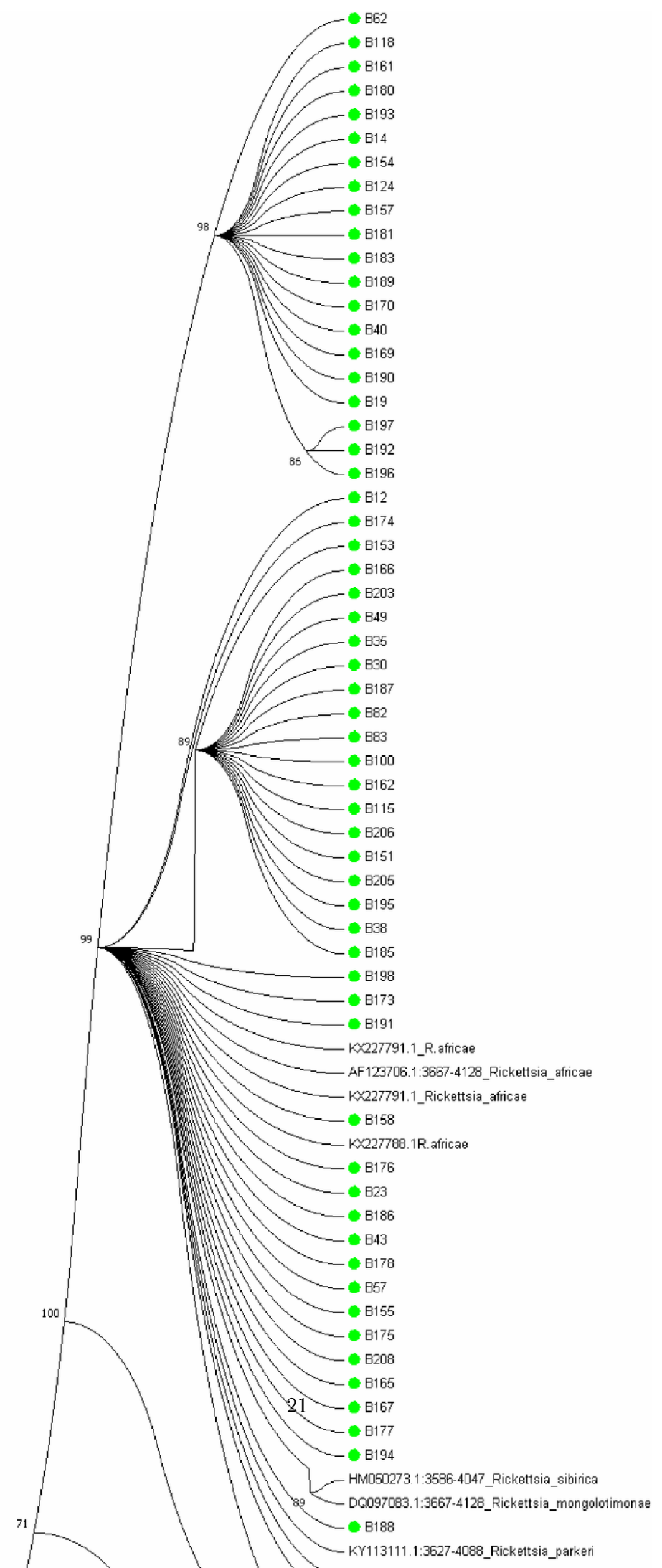

